\title{
Towards Agile DNN Accelerator Design Using Incremental Synthesis on FPGAs
}

\author{
Qingcheng Xiao \\ walkershaw@foxmail.com \\ Peking University \\ Beijing, China
}

\begin{abstract}
Hardware-software co-design is the new trend for deep neural network and FPGA accelerator development, which iteratively revises and tunes the full system. The bottleneck of the approach lies in the time-consuming hardware synthesis. In this paper, we propose an incremental synthesis framework Acoda to rapidly design DNN accelerators on FPGAs. Based on the observation that most revisions to DNNs are minor and local, Acoda reuses existing hardware modules and incrementally modifies the accelerator. It first detects the software revisions using a graph edit distance algorithm. Then, it maps the software revisions to hardware revisions through a multi-level reuse hierarchy. As a result, Acoda speeds up the design process by $9.31 \mathrm{X}$ to $34.17 \mathrm{X}$ and achieves performance results comparable to off-the-shelf accelerators.
\end{abstract}

\section{CCS CONCEPTS}

- Computer systems organization $\rightarrow$ Neural networks.

\section{KEYWORDS}

agile design; DNN accelerator; incremental synthesis

\section{ACM Reference Format:}

Qingcheng Xiao and Yun Liang. 2022. Towards Agile DNN Accelerator Design Using Incremental Synthesis on FPGAs. In Proceedings of the 2022 ACM/SIGDA International Symposium on Field-Programmable Gate Arrays (FPGA '22), February 27-March 1, 2022, Virtual Event, CA, USA. ACM, New York, NY, USA, 7 pages. https://doi.org/10.1145/3490422.3502351

\section{INTRODUCTION}

Deep neural networks (DNNs) have been applied among a wide range of domains. Hardware acceleration is a natural solution to the large computation requirement imposed by DNNs. Among commercial hardware platforms, FPGAs have become commonplace for prototyping accelerators as they provide high performance and energy efficiency $[2,14,15,24,25,28,31,32]$. More importantly, FPGA accelerators can also be co-designed with the DNNs in applicationspecific scenarios [1] as FPGAs are customizable. For instance, both

\footnotetext{
${ }^{*}$ Corresponding Author

Permission to make digital or hard copies of all or part of this work for personal or classroom use is granted without fee provided that copies are not made or distributed for profit or commercial advantage and that copies bear this notice and the full citation on the first page. Copyrights for components of this work owned by others than ACM must be honored. Abstracting with credit is permitted. To copy otherwise, or republish, to post on servers or to redistribute to lists, requires prior specific permission and/or a fee. Request permissions from permissions@acm.org.

FPGA '22, February 27-March 1, 2022, Virtual Event, CA, USA

(c) 2022 Association for Computing Machinery.

ACM ISBN 978-1-4503-9149-8/22/02 . \$ $\$ 15.00$

https://doi.org/10.1145/3490422.3502351
}

\author{
Yun Liang* \\ ericlyun@pku.edu.cn \\ Peking University \\ Beijing, China
}

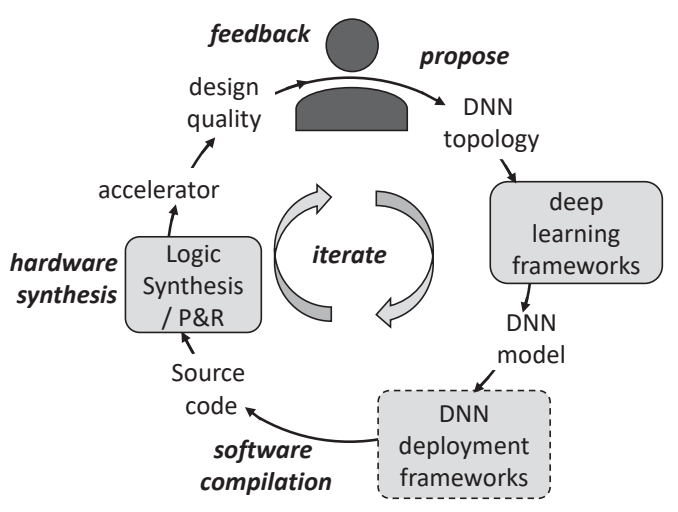

Figure 1: A typical design flow for DNN accelerators.

the accelerator and the DiracDeltaNet DNN proposed by [34] are tailored and optimized for classification accuracy and inference speed. The accelerators derived from the co-design process can customize modules for each DNN layer such that they are dedicated to the target DNNs. Compared with the general programmable DNN accelerators $[6,21]$, the co-design solution provides higher performance [29]. For example, co-design [34] yields a 16.9X frame rate improvement by optimizing the architecture and DNN structure (Conv-Pooling-Shift-Shuffle) in concert.

A typical design flow for DNN accelerators is illustrated in Figure 1. Users propose DNN topologies, train and deploy them on FPGAs with existing frameworks. The DNNs are executed on the accelerators to measure design quality. Based on the quality, the users will modify the DNN structure or tune some parameters. The process continues till the accelerators meet the users' requirement. The co-design flow involves two compilation steps: software compilation and hardware synthesis. The software compilation step will spend seconds or minutes in compiling the DNNs into design source code. The hardware synthesis step will synthesize the design source code into the final physical implementation of the hardware, which involves a series of lengthy passes on FPGAs, including HLS, logic synthesis, $\mathrm{P} \& \mathrm{R}$. The synthesis time depends on tools, design size, frequency, etc. HLS, logic synthesis, and $\mathrm{P} \& \mathrm{R}$ passes all take hours, making hardware synthesis orders of magnitude slower than the software compilation and the major hurdle of the co-design. However, in the current design flow, we have to go through the entire synthesis pass on FPGAs for even subtle changes to DNNs.

We observe that in the co-design flow, 1) most changes in DNN structures are local and minor, 2) these changes are often made one at a time. In particular, there are a few key DNN design knobs: feature map dimensions (number and size), operators, and network depth. For example, one can reduce the feature map number to 


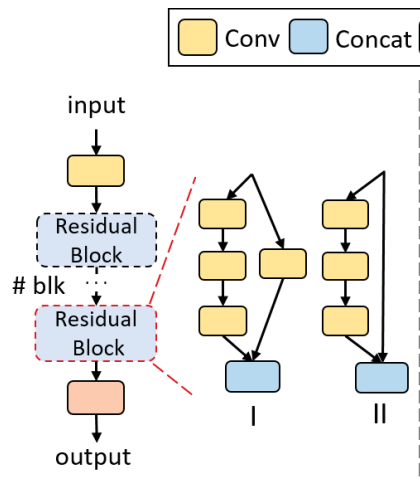

(a) ResNet

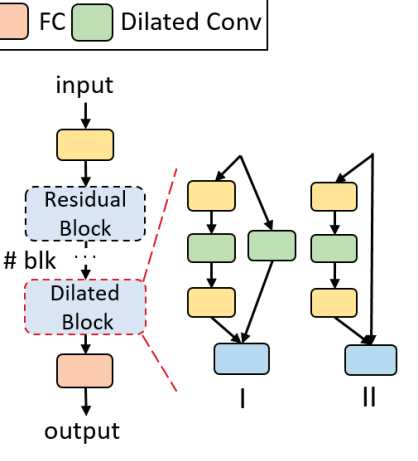

(b) DRN
Figure 2: DNN building blocks. blk: block.

lower the computation complexity. This motivates us to design an incremental synthesis flow specific for DNN design on FPGAs, where only the changes are re-synthesized. Incremental synthesis has been applied to multiple synthesis passes in the past. For timing analysis, [9] incrementally calculates timing in a netlist using the previous analysis results. For logic and physical synthesis, incremental flows [17] partition designs and individually synthesize, $\mathrm{P} \& \mathrm{R}$ each region.

To this end, we propose Acoda, an incremental synthesis flow for DNN design on FPGAs. Acoda can automatically detect common DNN revisions by calculating graph edit distance. Then, we propose a three-level reuse hierarchy based on DNN graph to reuse the hardware design as much as possible and synthesize the design incrementally. The three reuse levels include sub-graph reuse, node reuse, and partial reuse. The revised hardware modules will be re-implemented and integrated into the design, while the rest parts remain the same. To sum up, our key contributions are:

- We develop Acoda, an incremental synthesis framework for designing DNN accelerators on FPGAs. Acoda can effectively shorten the hardware development time in co-design processes.

- We identify software revision patterns and develop a graph algorithm to detect them from DNN graphs.

- We develop a reuse hierarchy with different levels of hardware reuse and synthesis overhead in response to the DNN revisions.

Experiments demonstrate that Acoda achieves 9.31X to 34.17X co-design time reduction for Xilinx FPGAs. Designs generated by Acoda offer comparable performance compared with off-the-shelf accelerators.

\section{BACKGROUND AND MOTIVATION}

\subsection{DNNs on FPGAs}

Various application-specific DNNs have been implemented on FPGAs. For instance, [31, 33] design accelerators for real-time object detection. [2] proposes an accelerator for object classification, which leverages the cross-layer shortcut in ResNet [7]. In general, these DNNs are often composed of commonly used patterns or structures. For instance, ResNet employs residual blocks as its building blocks. As shown in Figure 2(a), there are two types of residual blocks. The type II block directly concatenates its input features with the results after three convolutional layers, while the type I uses a convolutional layer to process the input features before the concatenation. Developers tend to duplicate the number of blocks to improve accuracy. Moreover, some new DNNs are just variants of a previous DNN. For example, both ResNeXt [30] and Dilated Residual Networks (DRN) [35] are changed from ResNet. To enlarge the receptive fields of output features, DRN slightly changes the building block, as shown in Figure $2($ b). It replaces $3 \times 3$ convolutions of 9 residual blocks with dilated convolutions.

In co-design processes, developers can tailor the computation of DNNs to match the compute capability of the target FPGAs. For instance, convolutions have several variants: dilated, shuffled, group, depthwise, and transposed convolution. They have different computation complexity and behave differently on the hardware. Developers can choose the convolution variant that performs the best on the target FPGA platform.

\subsection{DNN Accelerator Design}

A DNN accelerator usually consists of multiple hardware modules accelerating specific functions, such as convolutions and matrix multiplications. The accelerators can be built manually or automatically using deployment frameworks [3, 8, 10, 18-20, 22, 23], which automatically employ IPs and configure IP parameters.

However, existing frameworks [3, 8, 18-20, 23] have fundamental limitations in hardware synthesis, performance, and design cost. These frameworks do not facilitate any hardware reuse. They always output the source code of hardware designs, which need to go through the lengthy hardware synthesis flow every time. As a result, these frameworks synthesize the entire accelerator, even if there are only subtle changes to the deep neural net. In addition, the performance of the designs generated by these frameworks is not optimal. TVM and [8] rely on programmable accelerators and instruction sets. The instruction set offers substantial flexibility to varying DNN workloads but with extra overhead in performance and energy efficiency. DNNWEAVER [18] employs customized modules in its accelerator design. To this end, we propose our framework. For the first time, Acoda offers a fast and efficient framework for DNN accelerator design by identifying the minor and local changes in DNNs and reusing hardware implementations.

\section{ACODA}

Figure 3 presents an overview of Acoda. As shown, the only user input is a DNN algorithm, and the output is the final accelerator dedicated for the DNN. Acoda requires a reference run to implement the current run. Initially, it lowers the DNN into a computation graph where nodes are layers, and edges represent data dependency. Based on the graph, it builds a dedicated accelerator where each node of the graph is mapped to an individual hardware module. More clearly, as the limited hardware resources may not accommodate all the nodes in modern complex DNNs, Acoda partitions the graph into multiple sub-graphs. Then it generates an architecture for each sub-graph and dynamically switches architectures as a new sub-graph starts to execute. Within an architecture, each module is synthesized, optimized, placed and routed individually within a fixed location in the device. Acoda caches all the intermediate logic netlists and physical implementations for further reuse. In the paper, we refer to logic netlists as netlists for simplicity. 


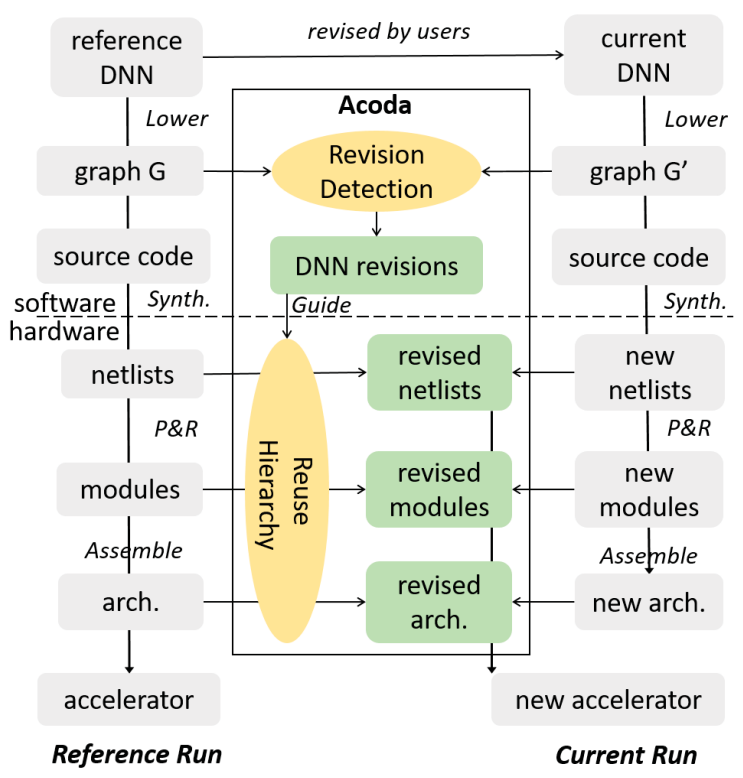

Figure 3: The workflow of Acoda.

With the previous run as the reference, Acoda implements the current run incrementally. As the right of Figure 3 shows, Acoda detects revisions between the current and the reference computation graphs (Section 4). The revisions then guide hardware revisions through a reuse hierarchy (Section 5), which handles revisions at different levels. When a sub-graph is unchanged, Acoda reuses the corresponding architecture's physical implementation without any modification. When a single node of a sub-graph is revised, Acoda can reuse hardware modules for other uninvolved nodes. The backend also explores parameters to build a new module for the revised node. If multiple nodes changes and the entire architecture requires re-implementation, Acoda backend reuses hardware modules partially. It reuses synthesized netlists as much as possible and integrates them with the newly generated modules, enabling rapid netlist assembling. With this reuse hierarchy, most parts of the reference designs can be reused to reduce the overall run time.

\section{DNN REVISIONS}

\subsection{Revision Patterns}

By analyzing the evolution of state-of-the-art DNNs from ResNet to DRN, we find that the revisions are minor and local in each codesign iteration. We identify seven common revision patterns, as shown in Table 1. Let the graphs of the reference run and the current run be $G=\langle N, E\rangle$ and $G^{\prime}=\left\langle N^{\prime}, E^{\prime}\right\rangle$, respectively, where $N\left(N^{\prime}\right)$ represents nodes and $E\left(E^{\prime}\right)$ represents edges. The patterns are:

(a) Insert a new node into the graph. It maps a null node $\varepsilon$ to a node $\mu \in N^{\prime}$.

(b) Remove maps a node $v \in N$ to a null node $\varepsilon$.

(c) Replace a node with another node that has different operations. It maps a node $v \in N$ to another node $\mu \in N^{\prime}$ if $\mu$ and $v$ have distinct types.

(d) Compress certain nodes. It shares the same notation with the replace pattern. However, it requires the pair of nodes have the same layer type but different parameters.
Table 1: Edit operation set. $\alpha, \beta$ : coefficients.

\begin{tabular}{|c|c|c|}
\hline Pattern & Notation & Cost Function \\
\hline Insert & $\varepsilon \rightarrow \mu$ & $\alpha \cdot O P s(\mu) / O P s_{\max }$ \\
\hline Remove & $v \rightarrow \varepsilon$ & $\alpha \cdot O P s(v) / O P s_{\max }$ \\
\hline Replace & $v \rightarrow \mu$ & $\begin{array}{c}\max \{O P s(v), O P s(\mu)\} \cdot \\
\beta / O P s_{\max }\end{array}$ \\
\hline Compress & $v \rightarrow \mu$ & $\|\vec{v}-\vec{\mu}\|$ \\
\hline Reorder & $\begin{array}{c}v_{1} \rightarrow \mu_{2} \& v_{2} \rightarrow \mu_{1}, \\
\left\langle v_{1}, v_{2}\right\rangle \in E\end{array}$ & Const. \\
\hline Decompose & $\begin{array}{c}\left\langle\left\langle\mu_{1}, \mu_{2}\right\rangle \in E^{\prime}\right. \\
\left(\mu_{1}, \ldots, \mu_{n}\right)\end{array}$ & $\begin{array}{c}\max \left\{O P s(v), \sum O P s(\mu)\right\} \cdot \\
\alpha / O P s_{\max }\end{array}$ \\
\hline Duplicate & - & - \\
\hline
\end{tabular}

(e) Reorder two neighboring nodes. It can be regarded as two edit operations $v_{1} \rightarrow \mu_{2}$ and $v_{2} \rightarrow \mu_{1}$, if two edges $\left\langle v_{1}, v_{2}\right\rangle$ and $\left\langle\mu_{1}, \mu_{2}\right\rangle$ exist in the graphs.

(f) Decompose is to substitute a node $v$ of $G$ with $n$ sequential nodes $\left(\mu_{1}, \ldots, \mu_{n}\right)$ of $G^{\prime}$.

(g) Duplicate is to insert a replica of a sub-graph into the graph. It cannot be succinctly denoted since both the involved node number and topology are uncertain.

\subsection{Revision Detection Algorithm}

The difference between the current and reference graphs can be regarded as a sequence of revision patterns. Multiple revision sequences exist for a pair of computation graphs. Acoda determines the sequence by calculating the approximate graph edit distance [16].

Graph edit distance is the total cost of revision patterns in a sequence. Specific to our problem, the revision patterns is the set of graph edit operations. We define the cost according to the synthesis time of each pattern, as listed in Table 1 . The synthesis time positively correlates with the occupied resource of involved nodes in a revision. The resource, in turn, is determined by the computation complexity of a node. In Table 1 , we use a feature vector $\vec{v}$ to represent the node $v$. The vector consists of the normalized computation complexity and layer parameters, including input shape, filer shape, stride, and the maximal operation numbers, etc. of node $v$. When inserting or removing a node, we set its weighted computation complexity as the cost. We calculate the weighted computation complexities before and after decomposition and assign the maximum as the cost of the decompose pattern. The cost of replacing the node $\mu$ with $v$ equals to the sum of their weighted computation complexity. However, we use a smaller weight coefficient here so that a replacement is prior to an insertion plus a removal. We define the compress pattern's cost as the Euclidean distance of the feature vectors of the paired nodes. We assign a constant cost to the reorder pattern. The duplicate revisions are handled by a post-process. After finding out all inserted nodes, we enumerate sub-graphs of $G^{\prime}$ composed of the inserted nodes. A duplication would be recognized if the composed sub-graph is isomorphic to any sub-graph of $G$.

The approximate edit distance algorithm with the defined edit operation set and cost functions has $O\left(|N|^{3}\right)$ time complexity. We also use the VF2 sub-graph isomorphism algorithm [4] to recognize duplications from inserted nodes, which has $O\left(|N|^{2}\right)$ time complexity. By finding out the minimal edit distance, we obtain a revision sequence, which be used to revise the accelerator design. 


\section{REUSE HIERARCHY}

\subsection{Underlying Architecture}

Acoda caters to application scenarios where users optimize a dedicated accelerator for one DNN. In our accelerator design, we employ a hardware module for each node in the computation graph [27]. We can deeply customize the module parameters and interconnection for each node. Such dedicated design can greatly improve performance and meet other design objectives, including accuracy, power, throughput, etc. This architectural design also enables hardware reuse as modules are decoupled. When certain modules require modifications, only these modules need to be resynthesized without impacting other modules.

\subsection{Methodology}

The revision patterns have different impacts on the hardware as they involve different numbers of nodes. We develop a three-level hierarchy to reuse the reference hardware design and synthesize the current design incrementally. The three reuse levels are 1) subgraph reuse: reusing the full physical implementation of a subgraph, 2) node reuse: reusing the full physical implementation of a node (layer) without the $P \& R, 3)$ partial reuse: reusing the netlist of a hardware module, but need to rerun the P\&R. Specifically, we handle the duplicate pattern at the sub-graph level if the duplicated nodes are from the same sub-graph. Insert, remove, and decompose patterns change the node number and topology of the sub-graph, calling for re-synthesizing the hardware design. Therefore, we only reuse the netlists of some unchanged modules. The reorder, replace, and compress patterns provide more reuse opportunities as they do not change the number of nodes. We directly reuse the implementations of the unchanged layers.

Figure 4 gives an example where we partition the computation graph into five sub-graphs and revise the second sub-graph. The fourth sub-graph is duplicated from the third sub-graph. We use this example to illustrate the reuse hierarchy.

Sub-graph Reuse. We use $A, B$, and $B^{\prime}$ to denote three subgraphs. $B^{\prime}$ is from the current DNN graph, while $A$ and $B$ are from the reference DNN graph. The sub-graph reuse relations are denoted as below:

$$
\operatorname{Arch}\left(B^{\prime}\right)= \begin{cases}\operatorname{Arch}(B), & B^{\prime}=B \\ \operatorname{Arch}(A), & B^{\prime}=A \\ \operatorname{Arch}(A) \cdot r m\left(A-B^{\prime}\right), & B^{\prime} \subset A\end{cases}
$$

where Arch represents the physical architecture implementation for a sub-graph, $r m$ is to remove modules from the implementation, $A-$ $B^{\prime}$ represents the nodes existing only in $A$. Naturally, if no revision is made to a sub-graph $\left(B^{\prime}=B\right)$, the corresponding architecture is directly reused. In Figure 4, Arch 1, 3, and 5 are in this case. If the new sub-graph $B^{\prime}$ is completely duplicated from $A\left(B^{\prime}=A\right)$, such as Arch 4 and Arch 3 in the figure, $A$ and $B^{\prime}$ will share the same physical implementation. In more general cases where a subgraph $B^{\prime}$ is duplicated from a part of $A$, Acoda completely duplicates $\operatorname{Arch}(A)$ and removes the redundant modules $\left(A-B^{\prime}\right)$.

Node Reuse. We observe that most reorder revisions happen to convolutional layers and their subsequent pooling layers. Bringing the pooling layer forward reduces the feature map size and

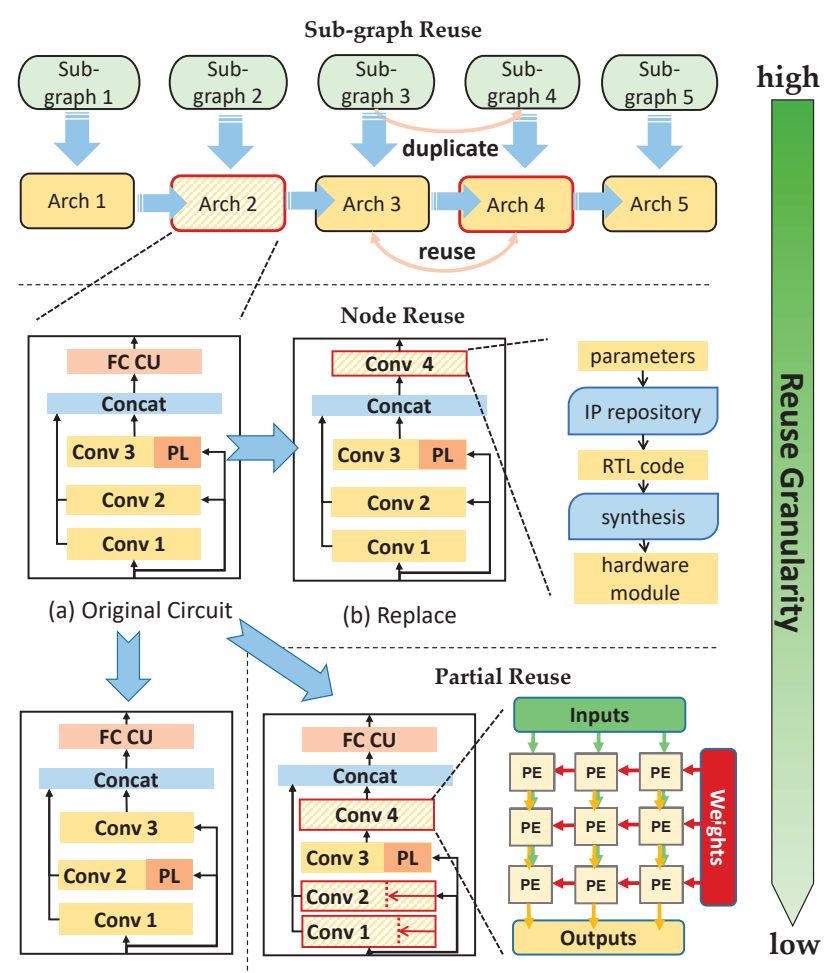

(c) Reorder

(d) Insert

(e) A conv module

Figure 4: Reuse hierarchy.

computations. Thus, we develop convolution modules embedding pooling functions. The pooling function is bypassed by default. In Figure 4(c), reordering Conv 3 and its subsequent pooling layer equals to enabling the pooling function of Conv 2. In this way, convolution modules are fully reused. For the replace pattern, Acoda will implement a new module with the replaced module's resource and location. Figure 4(b) gives an example where the fully connected module FC is replaced by Conv 4. Acoda reuses the implementation of all other modules except for FC.

Partial Reuse. Removing a node can be rapidly enabled by bypassing the associated module. However, when the bypassed modules occupy substantial resources, the accelerator performance would be low. To avoid this problem, Acoda re-implements the architecture for the sub-graph if the utilization is below a threshold. Insert and decompose revisions always change the sub-graph topology and introduce new modules to the architecture. Since existing modules have fixed physical locations and occupy the most resources, directly inserting new modules hardly meets resource constraints. Acoda would generate a new architecture for the revised sub-graph.

When re-implementing the architecture of a sub-graph, parameters of existing modules may or may not change. Acoda reuses the synthesized netlists of modules whose parameters are unchanged. These reused netlists avoid logic synthesis and can be placed and routed at a new location. Figure 4(d) illustrates the case where Conv 4 module is inserted into Arch 2. Conv 1 and Conv 2 modules are shrunken to make room for Conv 4. The netlists of Conv 3 and FC modules remain unchanged and are fully reused. 
For modules with changed parameters, Acoda will synthesize, place, route new modules and integrate them into the final design. Acoda can further speed up module synthesis by rapid netlist assembling. Specifically, in convolution modules and fully connected modules, Acoda connects their processing elements (PEs) in a mesh-like topology [12], as shown in Figure 4(e). These PEs are homogeneous and have identical netlists, and each outputs partial results. Acoda can reuse the netlist of one PE to build convolution or fully connected modules with arbitrary shapes.

\subsection{Implementation}

We use the RapidWright [13] tool to implement the reuse hierarchy. The partial reuse is to reuse logic netlists and the node reuse is to reuse physical netlists. We parse and edit logic netlists with read_edif, write_edif interfaces, and EDIFParser class. Modules are abstracted as EDIFNetlist objects. We remove and create EDIFNetlist objects to implement partial reuse. The RapidWright Module class contains both logical and physical netlists. We create and remove Module instances to achieve the node reuse. All revisions are saved as out-of-context design checkpoint files.

\section{EXPERIMENTS}

We define a co-design trace as a sequence of DNN topologies during co-design processes. In our experiments, we collect traces from YOLO, DRN, and neural architecture search (NAS). We use Acoda to incrementally generate accelerators for these traces and use Xilinx Vivado tools [13] for synthesis. We also compare Acoda-generated designs with previous YOLO accelerators. All the synthesis processes are performed on 2 Intel(R) Xeon(R) E5-2650 CPUs at 2.3GHz, with 64GB of DDR3 memory. We use a Xilinx ZCU102 FPGA board as the target hardware.

\subsection{YOLO Case Study}

YOLO is a real-time object detection CNN, which is proposed in 2016 and keeps evolving in recent years. Table 2 gives two versions of YOLO. As shown, there are 16 revisions between YOLO v1 and v2, including 1 decompose, 1 compress, 2 insert, 2 replace, 3 reorder, and 7 remove revisions. For instance, the $7 \times 7$ convolution conv 1 in YOLO v1 is decomposed into three layers in YOLO v2: two $3 \times 3$ convolutions and one maxpooling layer. We reproduce the evolution from YOLO v1 to v2 by revising the topology of YOLO v1 until it is identical to YOLO v2. Assume at most $\Delta$ revisions are made at each iteration. We collect 5 traces by varying $\Delta$ from 1 to 5 and refer to these traces as YOLO- $\Delta$. From the YOLO- 1 to the YOLO- 5 traces, $16,8,6,4$, and 4 iterations are taken, respectively.

Here, we take the YOLO- 2 trace as a case study to demonstrate the time saved by each reuse level. The YOLO-2 trace takes eight iterations and makes two sequential revisions at each iteration. We compare Acoda with the default Vivado synthesis flow, which uses the same sub-graph partitioning but synthesizes all architectures each time. Initially, Acoda partitions YOLO v1 into five sub-graphs shown in Table 2 and generates a reference design composed of five architectures. Then Acoda makes revisions to the reference design iteratively. Figure 5(a) shows the execution time comparison and Figure 5(b) shows the breakdown results. Except for the \#6 iteration, which involves two sub-graphs, all the other iterations involve only
Table 2: YOLO v1 and v2. Flt.: filter size. Str.: stride. Ch. I/O: input/output feature number.

\begin{tabular}{|c|c|c|c|c|c|c|c|c|c|}
\hline \multirow{2}{*}{$\begin{array}{l}\text { Sub- } \\
\text { graph }\end{array}$} & \multicolumn{4}{|c|}{ YOLO v1 } & \multicolumn{4}{|c|}{ YOLO v2 } & \multirow{2}{*}{$\begin{array}{c}\text { Revision } \\
\text { Pattern }\end{array}$} \\
\hline & Name & Flt. & Str. & Ch. I/O & Name & Flt. & Str & Ch. I/O & \\
\hline \multirow{9}{*}{1} & conv1 & $7 \times 7$ & 2 & $3 / 64$ & conv1 & $3 \times 3$ & 1 & $3 / 32$ & \multirow{9}{*}{$\begin{array}{c}\text { Decompose } \\
\\
\text { Insert } \\
\text { Reorder }\end{array}$} \\
\hline & \multirow{2}{*}{\multicolumn{4}{|c|}{ maxpooling }} & \multicolumn{4}{|c|}{ maxpooling } & \\
\hline & & & & & \multicolumn{4}{|c|}{ maxpooling } & \\
\hline & conv2 & $3 \times 3$ & 1 & $64 / 192$ & conv3 & $3 \times 3$ & 1 & $64 / 128$ & \\
\hline & & & & & conv4 & $1 \mathrm{x} 1$ & 1 & $128 / 64$ & \\
\hline & & $\max$ & oool & & conv5 & $3 \times 3$ & 1 & $64 / 128$ & \\
\hline & conv3 & $1 \mathrm{x} 1$ & 1 & $192 / 128$ & & $\max$ & ool & & \\
\hline & conv4 & $3 \times 3$ & 1 & $128 / 256$ & conv6 & $3 \times 3$ & 1 & $128 / 256$ & \\
\hline & conv5 & $1 \mathrm{x} 1$ & 1 & $256 / 256$ & conv7 & $1 \mathrm{x} 1$ & 1 & $256 / 128$ & \\
\hline \multirow{7}{*}{2} & \multirow{3}{*}{\multicolumn{4}{|c|}{$\begin{array}{llll}\text { conv6 } & 3 \times 3 & 1 & 256 / 512 \\
& \text { maxpooling }\end{array}$}} & conv8 & $3 \times 3$ & 1 & $128 / 256$ & \multirow{7}{*}{$\begin{array}{c}\text { Insert } \\
\text { Reorder }\end{array}$} \\
\hline & & & & & & $\max ]$ & ool & & \\
\hline & & & & & conv10 & $3 \times 3$ & 1 & $256 / 512$ & \\
\hline & conv7 & $1 \times 1$ & 1 & $512 / 256$ & conv11 & $1 \mathrm{x} 1$ & 1 & $512 / 256$ & \\
\hline & conv8 & $3 \times 3$ & 1 & $256 / 512$ & conv12 & $3 \times 3$ & 1 & $256 / 512$ & \\
\hline & conv9 & $1 \times 1$ & 1 & $512 / 256$ & conv13 & $1 \times 1$ & 1 & $512 / 256$ & \\
\hline & conv10 & $3 \times 3$ & 1 & $256 / 512$ & conv14 & $3 \times 3$ & 1 & $256 / 512$ & \\
\hline \multirow{5}{*}{3} & conv11 & $1 \times 1$ & 1 & $512 / 256$ & & & & & \multirow{5}{*}{ Remove } \\
\hline & conv12 & $3 \times 3$ & 1 & $256 / 512$ & & & & & \\
\hline & conv13 & $1 \mathrm{x} 1$ & 1 & $512 / 256$ & & & & & \\
\hline & conv14 & $3 \times 3$ & 1 & $256 / 512$ & & & & & \\
\hline & conv15 & $1 \times 1$ & 1 & $512 / 512$ & & & & & \\
\hline \multirow{5}{*}{4} & conv16 & $3 \times 3$ & 1 & $512 / 1024$ & \multicolumn{4}{|c|}{ maxpooling } & \multirow{5}{*}{ Reorder } \\
\hline & & $\max$ & ool & & conv15 & $3 \times 3$ & 1 & $512 / 1024$ & \\
\hline & conv17 & $1 \mathrm{x} 1$ & 1 & $1024 / 512$ & conv16 & $1 \mathrm{x} 1$ & 1 & $1024 / 512$ & \\
\hline & conv18 & $3 \times 3$ & 1 & $512 / 1024$ & conv17 & $3 \times 3$ & 1 & $512 / 1024$ & \\
\hline & conv19 & $1 \times 1$ & 1 & $1024 / 512$ & conv18 & $1 \times 1$ & 1 & $1024 / 512$ & \\
\hline \multirow{7}{*}{5} & conv20 & $3 \times 3$ & 1 & $512 / 1024$ & conv19 & $3 \times 3$ & 1 & $512 / 1024$ & \multirow{7}{*}{ Remove } \\
\hline & conv21 & $3 \times 3$ & 1 & $1024 / 1024$ & conv20 & $3 \times 3$ & 1 & $1024 / 1024$ & \\
\hline & conv22 & $3 \times 3$ & 2 & $1024 / 1024$ & & & & & \\
\hline & conv23 & $3 \times 3$ & 1 & $1024 / 1024$ & & & & & \\
\hline & conv24 & $3 \times 3$ & 1 & $1024 / 1024$ & conv21 & $3 \times 3$ & 1 & $1024 / 1024$ & \\
\hline & \multirow{2}{*}{\multicolumn{4}{|c|}{$\begin{array}{l}\text { fully-connected } \\
\text { fully-connected }\end{array}$}} & conv22 & $3 \times 3$ & 1 & $1536 / 1024$ & \\
\hline & & & & & conv23 & $1 \mathrm{x} 1$ & 1 & $1024 / 425$ & \\
\hline
\end{tabular}

one sub-graph. Acoda reuses the hardware implementation for the unchanged sub-graphs. As the figure shows, sub-graph reuse contributes the most to the overall time reduction.

In the \#1 iteration (decompose \& insert patterns) and \#3 iteration (insert \& reorder patterns), new layers are introduced into subgraph 1 and 2, respectively. The parameters of the corresponding architecture needs to be re-explored. In the \#2 iteration (reorder \& compress patterns) and \#8 iteration (two replace patterns), DNN revisions only affect several modules. Acoda merely implements new modules for the replaced layers. Node reuse contributes the synthesis time reduction of these two iterations. Acoda finds and handles similar revisions in other co-design iterations.

Acoda spends $15.8,15.29,12.8,9.2$, and 12.9 hours in synthesizing the YOLO- 1 to YOLO-5 traces, respectively. The default Vivado flow always synthesizes the five architectures during all iterations. For instance, it synthesizes 40 architectures in total for the YOLO-2 trace. It spends $280.47,142.36,88.74,67.75$, and 66.82 hours for the five traces, respectively. Overall, Acoda saves 4.83 days on average for these YOLO traces and achieves a 9.31X average time reduction.

\subsection{DRN Case Study}

ResNet-50 contains 4 type I residual blocks and 12 type II blocks. DRN replaces the convolutions in these residual blocks with dilated convolutions, leading to 9 replace revisions. We create DRN- $\Delta$ traces by varying the $\Delta$ value from 1 to 3 . It takes 9,5 , and 3 iterations for these traces, respectively. 


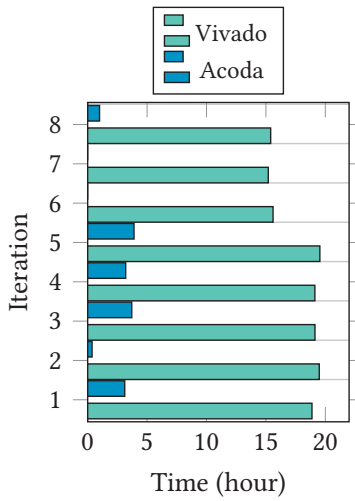

(a) Runtime comparison.

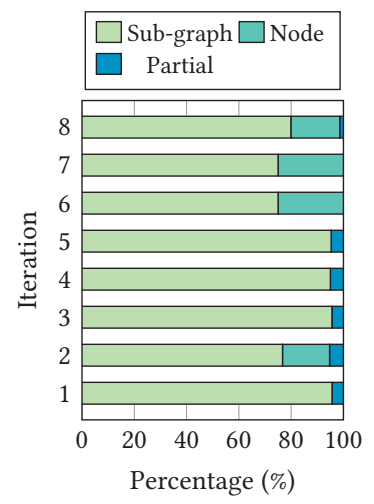

(b) Time reduction analysis.
Figure 5: Execution time comparison of the YOLO-2 trace.

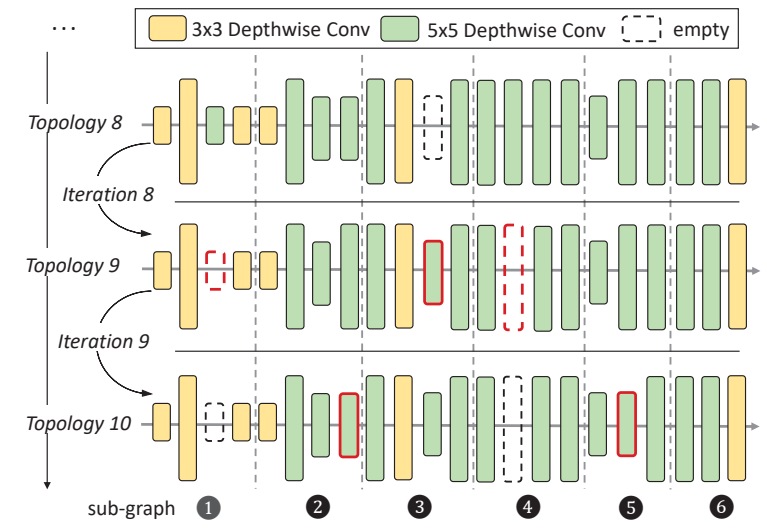

Figure 6: Three topologies of NAS-10. Each box denotes a block of [26]. The box size indicates the compute complexity.

Initially, Acoda partitions the reference ResNet-50 into eight subgraphs, and each includes two residual blocks. The DRN traces modify at most two sub-graphs at each iteration. Since all revisions are replacements, Acoda only needs to synthesize new dilated convolution modules at the replaced convolution modules' physical regions. It only takes 0.87 hours on average to implement each dilated convolution module. The Vivado flow spends around 11 days for these DRN traces. Overall, Acoda saves 10.84 days on average and achieves 34.17X speedup compared with the Vivado flow.

\subsection{NAS-based Sampling}

NAS is a machine-learning-based co-design technique. It searches DNN topologies within a given search space. More concretely, the search space used in this work specifies the maximal network depth (22 blocks) and candidate block types (9 types). All the candidate blocks consist of a $1 \times 1$ convolution, a depthwise convolution, and another $1 \times 1$ convolution. They differ in the filter size and computation complexity of the depthwise convolutions. At each iteration, NAS evaluates multiple DNN topologies. When targeting FPGAs, the DNN performance estimated by models [11] is often inaccurate, leading to a sub-optimal final topology. Acoda enables real evaluation on FPGAs by reducing the synthesis time. We randomly sample $\lambda=10$ intermediate topologies during the NAS process and refer to them as NAS-10. The trace consists of 11 replace, 2 insert, 2 remove, and 2 compress revisions.
Table 3: Comparisons of YOLO v2 accelerators.

\begin{tabular}{|c||c|c|c|c|}
\hline \multicolumn{1}{|c||}{ DNN } & \multicolumn{4}{c|}{ YOLO v2. Resolution: $416 \times 416}$. \\
\hline Framework & Acoda & {$[31]$} & {$[33]$} & cuDNN [5] \\
\hline Batch Size & 8 & 1 & 1 & 8 \\
\hline Platform & $\begin{array}{c}\text { Xilinx } \\
\text { ZCU102 }\end{array}$ & $\begin{array}{l}\text { Arria-10 } \\
\text { GX 1150 }\end{array}$ & $\begin{array}{l}\text { Arria-10 } \\
\text { GX 1150 }\end{array}$ & $\begin{array}{c}\text { NVIDIA } \\
\text { Jetson TX2 }\end{array}$ \\
\hline Precision & $\begin{array}{c}16 \text {-bit } \\
\text { fixed }\end{array}$ & $\begin{array}{l}\text { 8-bit } \\
\text { integer }\end{array}$ & $\begin{array}{l}16 \text {-bit } \\
\text { floating }\end{array}$ & $\begin{array}{c}16 \text {-bit } \\
\text { floating }\end{array}$ \\
\hline Frequency (GHz) & 0.2 & 0.2 & 0.278 & 1.3 \\
\hline Latency (ms) & 443.54 & 53.02 & 53.76 & 1607.38 \\
\hline Throughput (GOPS) & 541.1 & 566 & 558 & 149.31 \\
\hline Avg. Power (W) & 23.9 & 26 & 45 & 11.8 \\
\hline Efficiency (FPS/W) & 0.755 & 0.725 & 0.413 & 0.422 \\
\hline
\end{tabular}

In the NAS trace, sampled topologies have high similarity, which facilitates hardware reuse. The topology similarity is because NAS constrains both the network depth and layer types. To illustrate the NAS-10 trace clearly, we show the last two iterations of the trace in Figure 6. Acoda partitions these topologies into six subgraphs. At the \#8 iteration, one block is inserted in sub-graph 3, while two blocks are removed in sub-graph 1 and 4 . Architectures for the rest sub-graph 2, 5, and 6 can be reused at the sub-graph level. The removed block in sub-graph 1 occupies less than $15 \%$ hardware resources. The one in sub-graph 4 occupies less than $25 \%$ resources. Thus, Acoda directly bypasses the modules for these blocks instead of generating new architectures. For sub-graph 3, Acoda reallocates hardware resources to accommodate the inserted layer. At the \#9 iteration, one layer is compressed in sub-graph 2 , and another is compressed in sub-graph 5. Acoda re-explores parameters and implements new modules at the original resource regions. Overall, Acoda spends 0.65 days compared with 9.3 days of the default Vivado flow. We also sample 15 and 20 intermediate topologies to form two more NAS traces. Acoda achieve a 14.79X average time reduction.

\subsection{Performance Comparison}

Here, we also compare the design quality of Acoda-generated accelerator with a previous manually designed YOLO v2 accelerators and a TX2 GPU using cuDNN, as shown in Table 3. Our accelerator is obtained through the YOLO-2 co-design trace. Compared with previous works [31] and [33], our accelerator achieves similar throughput and even better energy efficiency. Compared with the TX2 GPU, we achieve 3.624X throughput/latency improvement and 1.836X efficiency improvement. Therefore, Acoda design flow provides both high performance and productivity.

\section{CONCLUSION}

The tedious and time-consuming hardware synthesis hinders the DNN and accelerator co-design. We propose Acoda for designing DNN accelerators on FPGAs rapidly. Acoda leverages different levels of hardware reuse to incrementally synthesize DNN accelerators. Experiments demonstrate that Acoda enables significant development time reduction and high design quality.

\section{ACKNOWLEDGMENTS}

This work is supported in part by National Natural Science Foundation of China (NSFC) under grant No.62090021. 


\section{REFERENCES}

[1] Mohamed S Abdelfattah, Łukasz Dudziak, Thomas Chau, Royson Lee, Hyeji Kim and Nicholas D Lane. 2020. Best of both worlds: Automl codesign of a cnn and its hardware accelerator. In 2020 57th ACM/IEEE Design Automation Conference (DAC). IEEE, 1-6.

[2] Arash Azizimazreah and Lizhong Chen. 2019. Shortcut Mining: Exploiting Crosslayer Shortcut Reuse in DCNN Accelerators. In 2019 IEEE International Symposium on High Performance Computer Architecture (HPCA). IEEE.

[3] Tianqi Chen, Thierry Moreau, Ziheng Jiang, Haichen Shen, Eddie Yan, Leyuan Wang, Yuwei Hu, Luis Ceze, Carlos Guestrin, and Arvind Krishnamurthy. 2018. TVM: End-to-End Optimization Stack for Deep Learning. In SysML Conference.

[4] Luigi P Cordella, Pasquale Foggia, Carlo Sansone, and Mario Vento. 2004. A (sub) graph isomorphism algorithm for matching large graphs. IEEE transactions on pattern analysis and machine intelligence (TPAMI) 26, 10 (2004), 1367-1372.

[5] NVIDIA Corporation. 2019. NVIDIA cuDNN. https://developer.nvidia.com/ cudnn

[6] Jeremy Fowers, Kalin Ovtcharov, Michael Papamichael, Todd Massengill, Ming Liu, Daniel Lo, Shlomi Alkalay, Michael Haselman, Logan Adams, Mahdi Ghandi, et al. 2018. A configurable cloud-scale DNN processor for real-time AI. In 2018 ACM/IEEE 45th Annual International Symposium on Computer Architecture (ISCA). IEEE, $1-14$.

[7] Kaiming He, Xiangyu Zhang, Shaoqing Ren, and Jian Sun. 2016. Deep residual learning for image recognition. In Proceedings of the IEEE conference on computer vision and pattern recognition (CVPR). 770-778.

[8] G. Hegde, Siddhartha, N. Ramasamy, and N. Kapre. 2016. CaffePresso: An optimized library for Deep Learning on embedded accelerator-based platforms. In 2016 International Conference on Compliers, Architectures, and Sythesis of Embedded Systems (CASES). 1-10.

[9] Tsung-Wei Huang, Guannan Guo, Chun-Xun Lin, and Martin DF Wong. 2020 Opentimer v2: A new parallel incremental timing analysis engine. IEEE Transactions on Computer-Aided Design of Integrated Circuits and Systems (TCAD) (2020).

[10] Liancheng Jia, Zizhang Luo, Liqiang Lu, and Yun Liang. 2021. TensorLib: A Spatial Accelerator Generation Framework for Tensor Algebra. In 2021 58th ACM/IEEE Design Automation Conference (DAC). 865-870. https://doi.org/10. 1109/DAC18074.2021.9586329

[11] Weiwen Jiang, Xinyi Zhang, Edwin H-M Sha, Lei Yang, Qingfeng Zhuge, Yiyu Shi, and Jingtong Hu. 2019. Accuracy vs. efficiency: Achieving both through fpgaimplementation aware neural architecture search. In 2017 54th Annual Design Automation Conference (DAC). 1-6.

[12] N. P. Jouppi, C. Young, N. Patil, D. Patterson, G. Agrawal, R. Bajwa, S. Bates, S Bhatia, N. Boden, A. Borchers, R. Boyle, P. Cantin, C. Chao, C. Clark, J. Coriell, M. Daley, M. Dau, J. Dean, B. Gelb, T. V. Ghaemmaghami, R. Gottipati, W. Gulland, R. Hagmann, C. R. Ho, D. Hogberg, J. Hu, R. Hundt, D. Hurt, J. Ibarz, A. Jaffey, A. Jaworski, A. Kaplan, H. Khaitan, D. Killebrew, A. Koch, N. Kumar, S. Lacy, J. Laudon, J. Law, D. Le, C. Leary, Z. Liu, K. Lucke, A. Lundin, G. MacKean, A. Maggiore, M. Mahony, K. Miller, R. Nagarajan, R. Narayanaswami, R. Ni, K. Nix, T. Norrie, M. Omernick, N. Penukonda, A. Phelps, J. Ross, M. Ross, A. Salek, E. Samadiani, C. Severn, G. Sizikov, M. Snelham, J. Souter, D. Steinberg, A. Swing, M. Tan, G. Thorson, B. Tian, H. Toma, E. Tuttle, V. Vasudevan, R. Walter, W. Wang, E. Wilcox, and D. H. Yoon. 2017. In-datacenter performance analysis of a tensor processing unit. In 2017 ACM/IEEE 44th Annual International Symposium on Computer Architecture (ISCA). 1-12.

[13] Chris Lavin and Alireza Kaviani. 2019. Build Your Own Domain-specific Solutions with RapidWright: Invited Tutorial. In Proceedings of the 2019 ACM/SIGDA International Symposium on Field-Programmable Gate Arrays (FPGA). ACM, 14-22.

[14] Yun Liang, Liqiang Lu, Qingcheng Xiao, and Shengen Yan. 2019. Evaluating Fast Algorithms for Convolutional Neural Networks on FPGAs. IEEE Transactions on Computer-Aided Design of Integrated Circuits and Systems (TCAD) (2019).

[15] Liqiang Lu, Jiaming Xie, Ruirui Huang, Jiansong Zhang, Wei Lin, and Yun Liang. 2019. An efficient hardware accelerator for sparse convolutional neural networks on FPGAs. In 2019 IEEE 27th Annual International Symposium on FieldProgrammable Custom Computing Machines (FCCM). IEEE, 17-25.

[16] Michel Neuhaus and Horst Bunke. 2004. An error-tolerant approximate matching algorithm for attributed planar graphs and its application to fingerprint classification. In foint IAPR International Workshops on Statistical Techniques in Pattern Recognition (SPR) and Structural and Syntactic Pattern Recognition (SSPR). Springer, 180-189.

[17] Rafael Trapani Possignolo and Jose Renau. 2019. SMatch: Structural matching for fast resynthesis in FPGAS. In 2019 56th Annual Design Automation Conference (DAC). 1-6.

[18] Hardik Sharma, Jongse Park, Divya Mahajan, Emmanuel Amaro, Joon Kyung Kim, Chenkai Shao, Asit Mishra, and Hadi Esmaeilzadeh. 2016. From high-level deep neural models to FPGAs. In 2016 49th Annual IEEE/ACM International Symposium on Microarchitecture (MICRO). IEEE, 1-12.

[19] Junzhong Shen, You Huang, Zelong Wang, Yuran Qiao, Mei Wen, and Chunyuan Zhang. 2018. Towards a Uniform Template-based Architecture for Accelerating
2D and 3D CNNs on FPGA. In Proceedings of the 2018 ACM/SIGDA International Symposium on Field-Programmable Gate Arrays (FPGA). ACM, 97-106.

[20] Yongming Shen, Michael Ferdman, and Peter Milder. 2017. Maximizing CNN accelerator efficiency through resource partitioning. In 2017 ACM/IEEE 44th Annual International Symposium on Computer Architecture (ISCA). IEEE, 535547

[21] Yongming Shen, Tianchu Ji, Michael Ferdman, and Peter Milder. 2019. Argus: An end-to-end framework for accelerating CNNs on FPGAs. IEEE Micro 39, 5 (2019), $17-25$.

[22] Anand Venkat, Tharindu Rusira, Raj Barik, Mary Hall, and Leonard Truong. 2019. SWIRL: High-performance many-core CPU code generation for deep neural networks. The International fournal of High Performance Computing Applications (HPCA) 33, 6 (2019), 1275-1289.

[23] Deguang Wang, Junzhong Shen, Mei Wen, and Chunyuan Zhang. 2019. An Efficient Design Flow for Accelerating Complicated-connected CNNs on a MultiFPGA Platform. In Proceedings of the 48th International Conference on Parallel Processing (ICPP). ACM, 98.

[24] Xuechao Wei, Yun Liang, and Jason Cong. 2019. Overcoming data transfer bottlenecks in FPGA-based DNN accelerators via layer conscious memory management. In 2019 56th ACM/IEEE Design Automation Conference (DAC). IEEE, 1-6.

[25] Xuechao Wei, Cody Hao Yu, Peng Zhang, Youxiang Chen, Yuxin Wang, Han $\mathrm{Hu}$, Yun Liang, and Jason Cong. 2017. Automated systolic array architecture synthesis for high throughput CNN inference on FPGAs. In 2017 54th Annual Design Automation Conference (DAC). 1-6.

[26] Bichen Wu, Xiaoliang Dai, Peizhao Zhang, Yanghan Wang, Fei Sun, Yiming Wu, Yuandong Tian, Peter Vajda, Yangqing Jia, and Kurt Keutzer. 2019. Fbnet: Hardware-aware efficient convnet design via differentiable neural architecture search. In Proceedings of the IEEE Conference on Computer Vision and Pattern Recognition (CVPR). 10734-10742.

[27] Qingcheng Xiao, Yun Liang, Liqiang Lu, Shengen Yan, and Yu-Wing Tai. 2017. Exploring heterogeneous algorithms for accelerating deep convolutional neural networks on FPGAs. In 2017 54th Annual Design Automation Conference (DAC).

[28] Oingcheng Xiao, Liqiang Lu, Jiaming Xie, and Yun Liang. 2020. FCNNLib: an efficient and flexible convolution algorithm library on FPGAs. In 2020 57th ACM/IEEE Design Automation Conference (DAC). IEEE, 1-6.

[29] Qingcheng Xiao, Size Zheng, Bingzhe Wu, Pengcheng Xu, Xuehai Qian, and Yun Liang. 2021. HASCO: Towards Agile HArdware and Software CO-design for Tensor Computation. In 2021 ACM/IEEE 48th Annual International Symposium on Computer Architecture (ISCA). 1055-1068. https://doi.org/10.1109/ISCA52012. 2021.00086

[30] Saining Xie, Ross Girshick, Piotr Dollár, Zhuowen Tu, and Kaiming He. 2017. Aggregated residual transformations for deep neural networks. In Proceedings of the IEEE conference on computer vision and pattern recognition (CVPR). 1492-1500.

[31] Ke Xu, Xiaoyun Wang, and Dong Wang. 2019. A scalable opencl-based fpga accelerator for yolov2. In 2019 IEEE 27th Annual International Symposium on Field-Programmable Custom Computing Machines (FCCM). IEEE, 317-317.

[32] Pengfei Xu, Xiaofan Zhang, Cong Hao, Yang Zhao, Yongan Zhang, Yue Wang, Chaojian Li, Zetong Guan, Deming Chen, and Yingyan Lin. 2020. AutoDNNchip: An automated dnn chip predictor and builder for both FPGAs and ASICs. In Proceedings of the 2020 ACM/SIGDA International Symposium on Field-Programmable Gate Arrays (FPGA). 40-50.

[33] Xianchao Xu and Brian Liu. 2018. FCLNN: a flexible framework for fast CNN prototyping on FPGA with OpenCL and Caffe. In 2018 International Conference on Field-Programmable Technology (FPT). IEEE, 238-241.

[34] Yifan Yang, Qijing Huang, Bichen Wu, Tianjun Zhang, Liang Ma, Giulio Gambardella, Michaela Blott, Luciano Lavagno, Kees Vissers, John Wawrzynek, and Kurt Keutzer. 2019. Synetgy: Algorithm-hardware Co-design for ConvNet Accelerators on Embedded FPGAs. In Proceedings of the 2019 ACM/SIGDA International Symposium on Field-Programmable Gate Arrays (FPGA). ACM, 23-32.

[35] Fisher Yu, Vladlen Koltun, and Thomas Funkhouser. 2017. Dilated residual networks. In Proceedings of the IEEE conference on computer vision and pattern recognition (CVPR). 472-480. 